\title{
Nano-Scaled Bionic Periosteum Orchestrating Osteogenic Microenvironment for Sequential Bone Regeneration
}

\author{
Hanwen Li ${ }^{1}$, , Huan Wang ${ }^{2, \#}$, Jun Pan ${ }^{1, \#, ~ J i a y i n g ~} \mathrm{Li}^{2}$, Kai Zhang ${ }^{1}$, Weifeng Duan ${ }^{1}$, \\ Huan Liang ${ }^{3}$, Kangwu Chen ${ }^{1}$, Dechun Geng ${ }^{1}$, Qin Shi ${ }^{1,2}$, Huilin Yang ${ }^{1,2, *}$, \\ Bin $\mathrm{Li}^{2}$, * , Hao Chen ${ }^{1,3 *}$
}

1 Department of Orthopedic Surgery, The First Affiliated Hospital of Soochow University, 899 Pinghai Road, Suzhou, Jiangsu, 215000, P. R. China.

2 Orthopedic Institute, Medical College, Soochow University, 708 Renmin Road, Suzhou, Jiangsu, 215000, P. R. China.

3 Medical College, Yangzhou University, 136 Jiangyang Road, Yangzhou, Jiangsu 225009, P. R. China.

${ }^{\#}$ These authors contributed equally to this word.

* Correspondence should be addressed to:

Dr. Hao Chen. Email: haochen@suda.edu.cn

Department of Orthopedics, The First Affiliated Hospital of Soochow University, 899 Pinghai Road, Suzhou, Jiangsu, 215000, P. R. China.

Prof. Bin Li. Email: binli@suda.edu.cn

Orthopedic Institution, Soochow University, 708 Renmin Road, Suzhou, Jiangsu, 215000, P. R. China.

Prof. Huilin Yang. Email: suzhouspine@163.com

Department of Orthopedics, The First Affiliated Hospital of Soochow University, 899 Pinghai Road, Suzhou, Jiangsu, 215000, P. R. China. 


\section{Supporting Information}

\section{Figure S1}

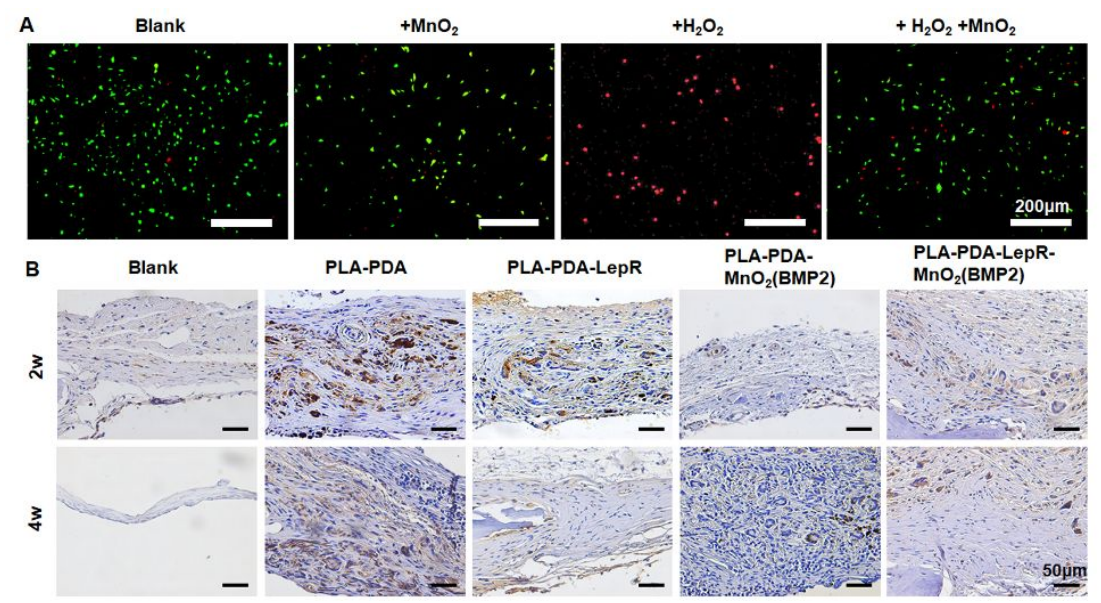

Figure S1. Anti-inflammation effect of $\mathbf{h}-\mathrm{MnO}_{2}$ nanoparticles. A. Live/dead staining of BMSCs treated with $400 \mu \mathrm{M} \mathrm{H}_{2} \mathrm{O}_{2}$ or $0.1 \mathrm{mg} / \mathrm{ml}$ h-MnO2 nanoparticles or both. B. Representative images of cyclooxygenase-2 (COX2) immunohistochemistory staining with the scaffolds collected from 2 weeks or 4 weeks cranial defect models.

\section{Figure S2}

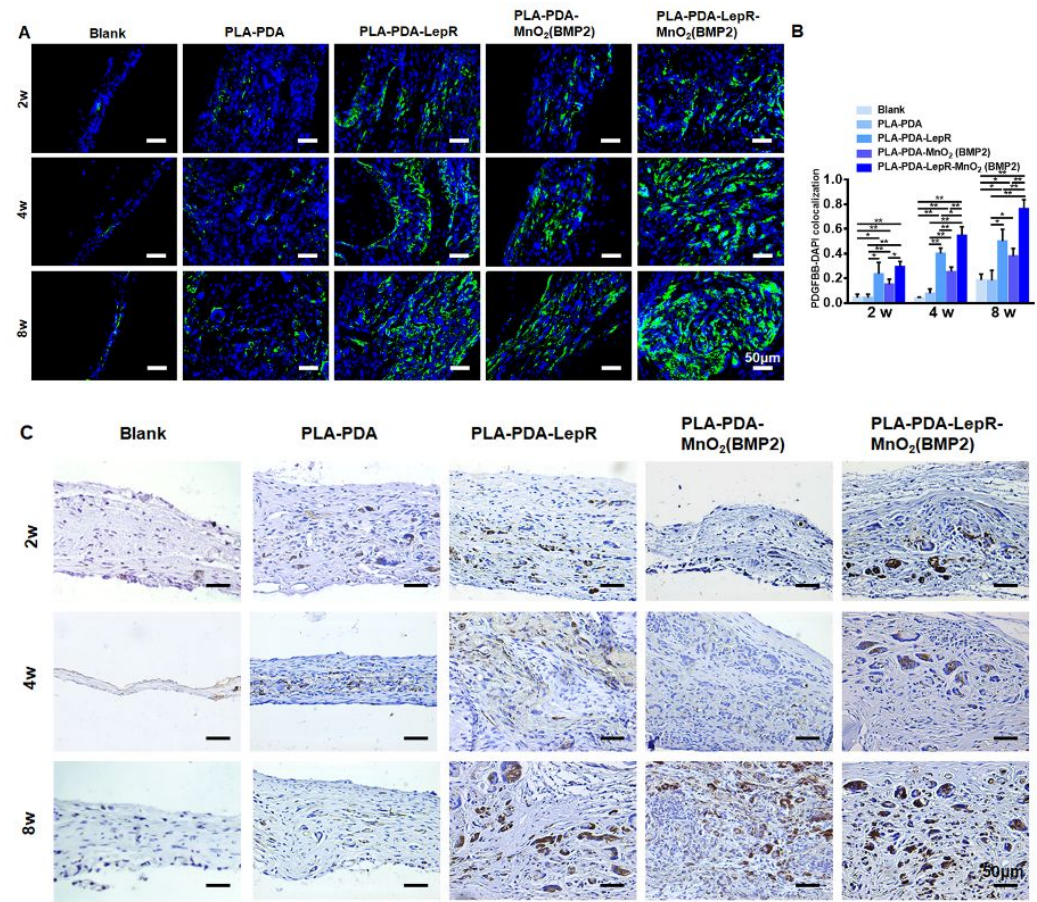

Figure S2. A. Representative images of PDGF-BB (green) staining in scaffolds from different groups. In all immunofluorescence staining, DAPI (blue) was used as a nuclear counterstain; B. Quantification of PDGF-BB staining. C. Immunohistochemical staining of HIF-1 in scaffolds from different groups. ANOVA test was applied for statistical analysis. ${ }^{*}$ stands for $p<0.05, * *$ stands for $p<0.01$ 